\title{
CANcer-specific Evaluation System (CANES): a high-accuracy platform, for preclinical single/multi-biomarker discovery
}

\author{
Min-Seok Kwon ${ }^{1}$, Seungyoon Nam $^{2,3,4}$, Sungyoung Lee ${ }^{1}$, Young Zoo Ahn ${ }^{5}$, Hae \\ Ryung Chang ${ }^{6}$, Yon Hui Kim 7 and Taesung Park ${ }^{1,8}$ \\ ${ }^{1}$ Interdisciplinary Program in Bioinformatics, Seoul National University, Seoul 08826, Korea \\ ${ }^{2}$ Gachon Institute of Genome Medicine and Science, Gachon University Gil Medical Center, Incheon 21565, Korea \\ ${ }^{3}$ Department of Life Sciences, Gachon University, Seongnam-si 13120, Korea \\ ${ }^{4}$ College of Medicine, Gachon University, Incheon 21565, Korea \\ ${ }^{5}$ System Cancer Science, Graduate School of Cancer Science and Policy, National Cancer Center of Korea, Goyang-si 10408, \\ Korea \\ ${ }^{6}$ Research Institute of Women's Health, Sookmyung Women's University, Seoul, 04310, Korea \\ ${ }^{7}$ Corestem Inc., Seongnam-si 13486, Korea \\ ${ }^{8}$ Department of Statistics, Seoul National University, Seoul 08826, Korea \\ Correspondence to: Taesung Park, email: tspark@stats.snu.ac.kr \\ Yon Hui Kim, email: yhkim@corestem.com
}

Keywords: biomarker performance, claudin, gastric cancer, transcriptome, data mining

Received: December 10,2016 Accepted: May 22, $2017 \quad$ Published: July 15, 2017

Copyright: Kwon et al. This is an open-access article distributed under the terms of the Creative Commons Attribution License 3.0 (CC BY 3.0), which permits unrestricted use, distribution, and reproduction in any medium, provided the original author and source are credited.

\section{ABSTRACT}

The recent creation of enormous, cancer-related "Big Data" public depositories represents a powerful means for understanding tumorigenesis. However, a consistently accurate system for clinically evaluating single/multi-biomarkers remains lacking, and it has been asserted that oft-failed clinical advancement of biomarkers occurs within the very early stages of biomarker assessment. To address these challenges, we developed a clinically testable, web-based tool, CANcer-specific single/multi-biomarker Evaluation System (CANES), to evaluate biomarker effectiveness, across 2,134 whole transcriptome datasets, from 94,147 biological samples (from 18 tumor types). For user-provided single/multi-biomarkers, CANES evaluates the performance of single/ multi-biomarker candidates, based on four classification methods, support vector machine, random forest, neural networks, and classification and regression trees. In addition, CANES offers several advantages over earlier analysis tools, including: 1) survival analysis; 2) evaluation of mature miRNAs as markers for user-defined diagnostic or prognostic purposes; and 3) provision of a "pan-cancer" summary view, based on each single marker. We believe that such "landscape" evaluation of single/ multi-biomarkers, for diagnostic therapeutic/prognostic decision-making, will be highly valuable for the discovery and "repurposing" of existing biomarkers (and their specific targeted therapies), leading to improved patient therapeutic stratification, a key component of targeted therapy success for the avoidance of therapy resistance.

\section{INTRODUCTION}

Traditionally, biomarker studies begin from a handful number of candidate genes or proteins, based on experimental and computational assessment. Also, for given candidates, validation techniques, and their supporting evidence, have been compromised, due to a lack of technical advances and publicly available clinical data. Now that various technologies, including nextgeneration sequencing, are mature, it is possibly to rapidly 
analyze "Big Data" (e.g., whole tumor transcriptomes and genomes) for association with clinical information. However, while these high-technology approaches should empower clinical researchers to identify clinical, translational, and accessible biomarkers, few approaches for this purpose have been successful [1]. To overcome the challenges of biomarker-driven cancer therapy, various standards and guidelines have been made to increase the rigor of the development process [2]. For screening purposes, diagnostic biomarkers would require a generally agreed upon requirement of high specificity and sensitivity, to allow general population screening for even the most common cancers [3]. For example, it is estimated that for a relatively rare disease such as ovarian cancer (1.3\% lifetime risk), effective (i.e., resulting in reduced mortality) screening, for an asymptomatic population of 2500 women, would require a sensitivity $\geq 75 \%$ and a specificity $\geq 99.6 \%$, to achieve a positive predictive value of $10 \%$ for the detection of individuals with stage 1 disease (at which the disease is $>90 \%$ curable) [4]. To achieve such predictive accuracy, it has been asserted that combinations of biomarkers ("biomarker panels") may allow obtainment of such stringent criteria $[5,6]$.

There are more than 200 types of cancer from over 60 different organs in the body [7]. Some cancers of different organs have many shared features, such as therapeutic response, while conversely, some cancer subtypes from the same organ are quite distinct [8]. These phenotypic features of cancer types depend on the expression patterns of single or multiple genes $[9,10]$. For example, since the oncogene ERBB2 (HER2) is amplified in subgroups of glioblastoma and, stomach, uterine, bladder, and lung cancers, responsiveness to HER2targeted therapy may or may not be analogous to that of HER2-amplified breast cancer $[9,10]$. Similarly, erlotinib, an effective inhibitor of the actively mutated epidermal growth factor receptor (EGFR), originally approved for the treatment of advanced pancreatic cancer, has now shown efficacy for non-small cell lung and various other cancers [11]. Here, to more rapidly make such preliminary determinations, we designed and developed a comprehensive web-based assessment tool, "CANcerspecific Evaluation System" (CANES), for exhaustive biomarker evaluation that: (i) employs repositories across 2,134 whole transcriptome datasets, from 94,147 biological samples (cell lines and normal and cancerous tissues), representing 18 tumor types; (ii) performs the initial steps of evaluating single and/or multi-genes as biomarker candidates; and (iii) uses various classification methods to support diagnostic or prognostic assessment of genes, as well as miRNAs, as biomarkers, yielding a "pan-cancer" summary view of the evaluation of each individual biomarker. Finally, one of the outstanding features of CANES is that it allows direct comparison between the diagnostic or prognostic performance of single vs. multi-biomarker sets. Multi-biomarker sets often tend to show good performance, by chance, when the number of biomarkers is large and sample size is small, resulting in artifactual results. CANES addresses this problem by providing standardized evaluation measures and empirical p-values, allowing direct comparison of the diagnostic/prognostic performance of multi-biomarker sets, having different numbers of biomarkers.

In summary, CANES represents a powerful tool for "landscape" evaluation across 18 cancer-types for single/multi-biomarkers, in association with diagnostic therapeutic decision making and prognostic use by preclinical researchers, producing high-quality results that can be further translated toward clinical "precision medicine."

\section{RESULTS}

\section{Demonstration of CANES performance in predicting single vs. multi-biomarker evaluation}

In our previous study, we identified several pathways involved in gastric cancer progression using our systems biology approach, PATHOME [12]. We also showed the significance of regulation of $\mathrm{HNF} 4 \alpha$, as well as reduced HIF $1 \alpha$, in early gastric cancer (GC) $[12,13]$, as detected only by our PATHOME algorithm (Figure 1A). We further found the HIF1-related pathway to associate with three claudin protein family members (claudins-1, -4 , and -18), by a protein-protein interaction tool, STRING (Search Tool for the Retrieval of Interacting Genes/Proteins, version 9.1) [14] (Figure 1A). Of the three claudins we identified to interact with an HIF-1 network, CLDN1 and $C L D N 4$ were previously reported as upregulated in gastric cancer progression, while $C L D N 18$ was downregulated [15-20]. Our CANES results were consistent with those previously shown gene expression patterns in another GC dataset (GSE13911) (Figure 1B). Table 1 shows that for biomarker use of the three $C L D N$ genes for gastric cancer, $C L D N 18$ had the highest balanced accuracy (BA), followed by $C L D N 1$ and then $C L D N 4$.

We next used CANES to predict each of the three $C L D N$ genes' ability to distinguish specific cancers among a panel of 18 tumor types. Figure 2 depicts radial plots (left panel) that represent four performance measures (area under curve; AUC, BA, sensitivity; SN, and specificity; SP) across 18 tumor types per single and/or multiple gene(s). All three claudin genes showed different predictive patterns. AUC plots demonstrated that $C L D N 1$ and $C L D N 18$ represent potential predictors of thyroid cancer, and CLDN4 and CLDN18, predictors of pancreatic cancer. Pairwise $C L D N$ biomarker AUC comparisons (heatmap, Figure 2, right panel), across 12 tumor types (The Cancer Genome Atlas; TCGA data), showed that $C L D N 1$ could readily distinguish colon from kidney, brain, lung, and ovary cancers, while both CLDN1 and CLDN4 (but not $C L D N 18$ ) could distinguish brain from kidney 
cancer. While these AUC values would not be sufficiently predictive to discriminate between specific tumor types, it is quite possible that their combination with other highly predictive markers or diagnostic methodologies (e.g., MRI, CT) could reach positive predictive values (PPVs) acceptable for early detection $[5,6]$.

Based on that (multiple biomarker) hypothesis, we evaluated the three claudin family genes as a multimarker. Figure 3A shows the multi-marker performances of CLDN1, CLDN4, and CLDN18 expression in distinguishing 18 tumor types. Figure $3 \mathrm{~B}-3 \mathrm{E}$ show multimarker performances in GC. Figure 3C shows higher values for the three-gene set, as compared to single marker performance in GC (Table 1). When CLDN1, CLDN4, and $C L D N 18$ were analyzed, as single markers, across 18 cancer tissue types, the AUC values were $0.756\left(\mathrm{p}=1.4 \times 10^{-}\right.$ $\left.{ }^{4}\right), 0.647(\mathrm{p}=0.156)$, and $0.792\left(\mathrm{p}=2.5 \times 10^{-4}\right)$, respectively. When CLDN1, CLDN4, and CLDN18 were analyzed throughout the 18 cancer types as a multi-marker set, the AUC value was $0.850\left(p=3.3 \times 10^{-4}\right)$ (Table 1 and Figure 4). Thus, these findings support the many assertions that multiple biomarker sets hold greater sensitivity/specificity, compared to single markers, for disease detection $[5,6]$, in general or at-risk populations. To address the problem that a randomly chosen marker set with a large number of probes often tends to show good performance, CANES provides empirical p-values.

In addition to evaluating biomarker performance for specific tumors, another key feature of CANES is its assessment of the predictive accuracy of multiple marker panels, among multiple cancer types. Consequently, we evaluated a panel of well-known breast cancer markers

A

HIF1- related pathway

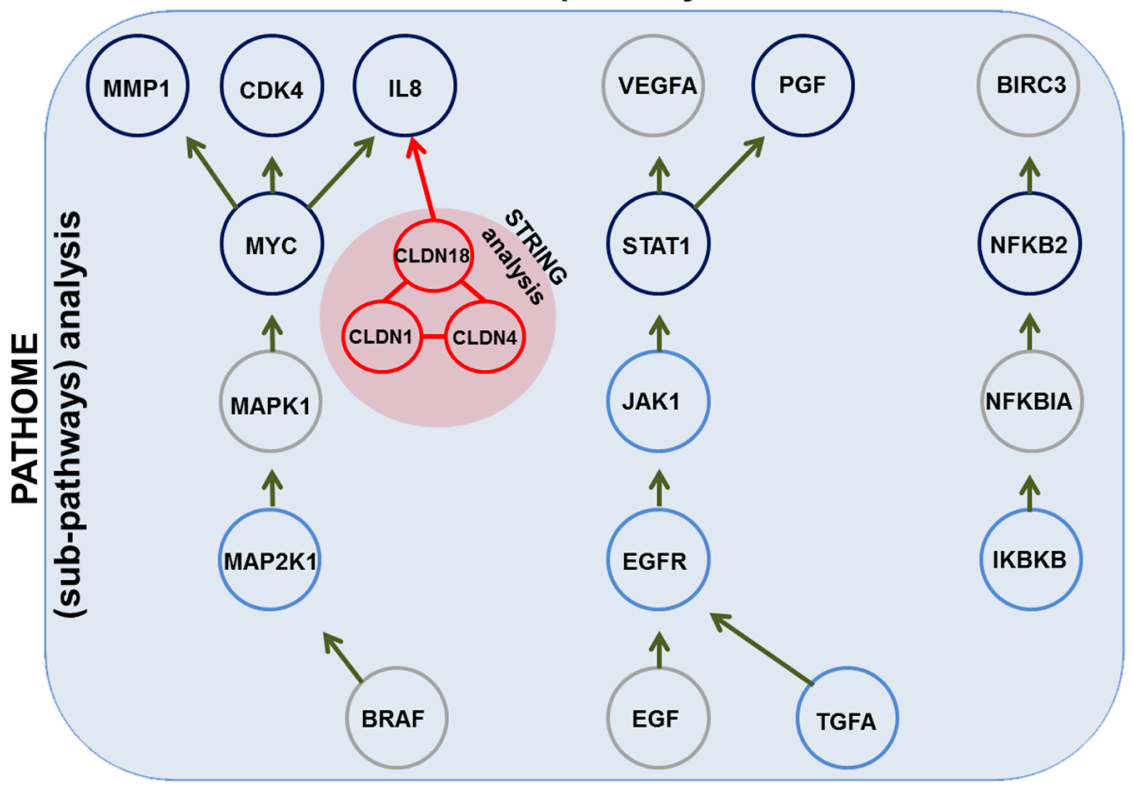

B

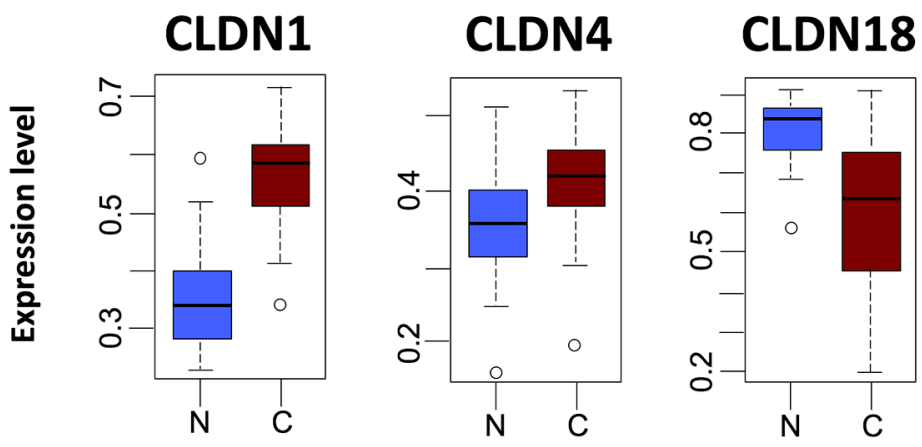

Figure 1: The figure here depicts HIF-1 pathway and gene expression levels of CLDN1, CLDN4, and CLDN18 in gastric cancer. (A) HIF-1-related pathway (previously identified by our established algorithm (12) showing additional interactions with a triad consisting of CLDN1, CLDN4, and CLDN18, as identified by the STRING database (indicated in the red-filled circle). (B) Gene expression levels of $C L D N 1, C L D N 4$, and $C L D N 18$, in gastric cancer ("C") vs. normal ("N") samples in a gastric cancer dataset (GSE13911). CLDN1 and $C L D N 4$ were upregulated in tumors, while CLDN18 was downregulated. 
Table 1: Prediction measures of three single markers (CLDN1, CLDN4, and CLDN18) and a multi-marker (CLDN1/4/18) for gastric cancer (cancer tissue vs. normal tissue)

\begin{tabular}{lcccc}
\hline Average evaluation measure & \multicolumn{3}{c}{ Single marker } & Multi-marker \\
\cline { 2 - 5 } & CLDN1 & CLDN4 & CLDN18 & (CLDN1, 4, 18) \\
\hline Area under curve (AUC) & 0.756 & 0.647 & 0.792 & 0.850 \\
Balanced accuracy (BA) & 0.776 & 0.659 & 0.801 & 0.851 \\
Accuracy (AC) & 0.892 & 0.784 & 0.905 & 0.936 \\
Sensitivity (SN) & 0.817 & 0.705 & 0.880 & 0.849 \\
Specificity (SP) & 0.696 & 0.590 & 0.705 & 0.851 \\
Positive predictive value (PPV) & 0.821 & 0.693 & 0.778 & 0.859 \\
Negative predictive value (NPV) & 0.618 & 0.548 & 0.855 & 0.851 \\
False positive rate (FPR) & 0.304 & 0.409 & 0.295 & 0.149 \\
False discovery rate (FDR) & 0.178 & 0.306 & 0.221 & 0.150 \\
F1 score (F1) & 0.789 & 0.690 & 0.822 & 0.849 \\
\hline
\end{tabular}

These performance measures are for prediction of cancer and normal in stomach cancer.

BRCA1, BRCA2, BRIP1, CHEK2, PALB2, RB1 [21], and TP53 [22], for predictive accuracy in lung cancer. Figure 5 shows the CANES evaluation report for those seven grouped breast cancer biomarkers, as classified by support vector machine and leave-one-out crossvalidation, using lung cancer datasets from 46 cancer and 45 normal tissues [23] as the testing dataset. Figure 5 shows a representative CANES performance report on the test dataset. The seven multi-markers (Figure 5A) were evaluated in multiple cancer types (Figure 5B), and showed higher AUC, BA, SN, and SP values for lung cancer (Figure 5C). However, this multi-marker set was not statistically significant $(\mathrm{p}=0.129$ for $\mathrm{BA}$ and $\mathrm{p}=0.156$ for AUC) in lung cancer. To find significant multi-marker sets, all possible subgroups of the seven genes were evaluated using the same lung cancer dataset. Finally, 10 subgroups had significant BAs and AUCs, among which a multi-marker set with BRIP1-RB1 showed the best performance, with a $\mathrm{BA}=0.9780(\mathrm{p}=0.009)$ and $\mathrm{AUC}=0.9995(\mathrm{p}=0.001)$ (Figure 5D). After adjusting for multiple testing, using the Westfall and Young multiple correction method [24], the adjusted p-values were 0.055 and 0.024 for BA and AUC, respectively. Based on this performance evaluation, these two biomarkers could potentially be applied to lung cancerdiagnostic evaluations, similar to a previous report that in addition to breast cancer, the oncogene ERBB2-HER2 is amplified in subgroups of glioblastomas and stomach, uterine, bladder, and lung cancers (thus suggesting possible repurposing of the anti-HER2 antibody trastuzumab for these cancers) [10]. This result demonstrates that transcriptomic analysis of molecular patterns across cancer types allows the etiologic and therapeutic knowledge of one cancer type to be applied to another, suggesting that therapy guidance/response markers for one tumor may also be appropriate for others. Therefore, CANES provides powerful prediction to evaluate biomarkers across cancer types.

In addition to the above, we previously reported five genes, ENAH, RAD51, CHEK2, ATF4, and ICOSLG, as possible drug response biomarkers in breast cancer [25]. Setting these genes as a reference set in breast cancer, we compared biomarker suggestion results for the five candidates by using each tool (Table 2). One widely used commercial tool, Ingenuity Pathway Analysis (IPA), does not report a numerical representation for performance evaluation, including AUC, except either detection or no detection for single genes [26]. Also, IPA cannot perform multi-gene biomarker performance evaluation, and relies on its own database [27]. Similarly, Oncomine merely reports limited quantified information, such as the number of significant differential analyses (driven by Student t-tests for two classes) relating to each candidate biomarker [28]. However, Oncomine does not describe evaluation quantification for multi-gene biomarker performance, and is restricted to microarray analysis [29]. Unlike these two tools, CANES reports diverse performance evaluations (only AUC shown due to limited space in Table 2) for multi-gene biomarkers, as well as for each candidate. For example, considering that AUC $>0.75$ supports good biomarker feasibility, ENAH and RAD51 could be repurposed for breast cancer diagnosis usage. Thus, CANES can introduce biomarker candidates from published literature for diagnosis of other cancer types, based on evidence-based measurements. 


\section{Dynamic usage of big-data-based for predicting mutli-biomarker system for oncology therapeutic development}

The utility of CANES extends beyond the above illustrations. For example, CANES supports the feasibility (e.g., high sensitivity and specificity) of using specific panels of biomarkers for widespread population screening for distinct cancer types (or at minimum, to individuals already at increased risk for such cancer types), representing the achievement of a previously extremely difficult endeavour [30, 31]. We concede that biomarker discovery using CANES represents merely one step in a long and arduous process $[2,32]$, according to the recently adopted REporting recommendations for tumor MARKers
(REMARK) guidelines [2]. However, should the newly discovered, tumor-specific gene expression biomarkers prove present in body fluids, improved preclinical accuracy could potentially enhance the eventual translation of such diagnostics [3] toward the long-desired goal of simple blood or urine tests for cancer detection in high-risk populations [33, 34]. Moreover, from a research perspective, identifying a strong association of a specific gene(s) with a particular tumor could facilitate understanding of the mechanism-ofaction(s) of that specific biomarker(s), and the identification of other druggable targets/pathways involved in the progression of that distinct tumor.

Thus, CANES represents a novel and publically available tool for enhancing the characterization/discovery of single/multi-biomarker sets for specific cancer types. This tool
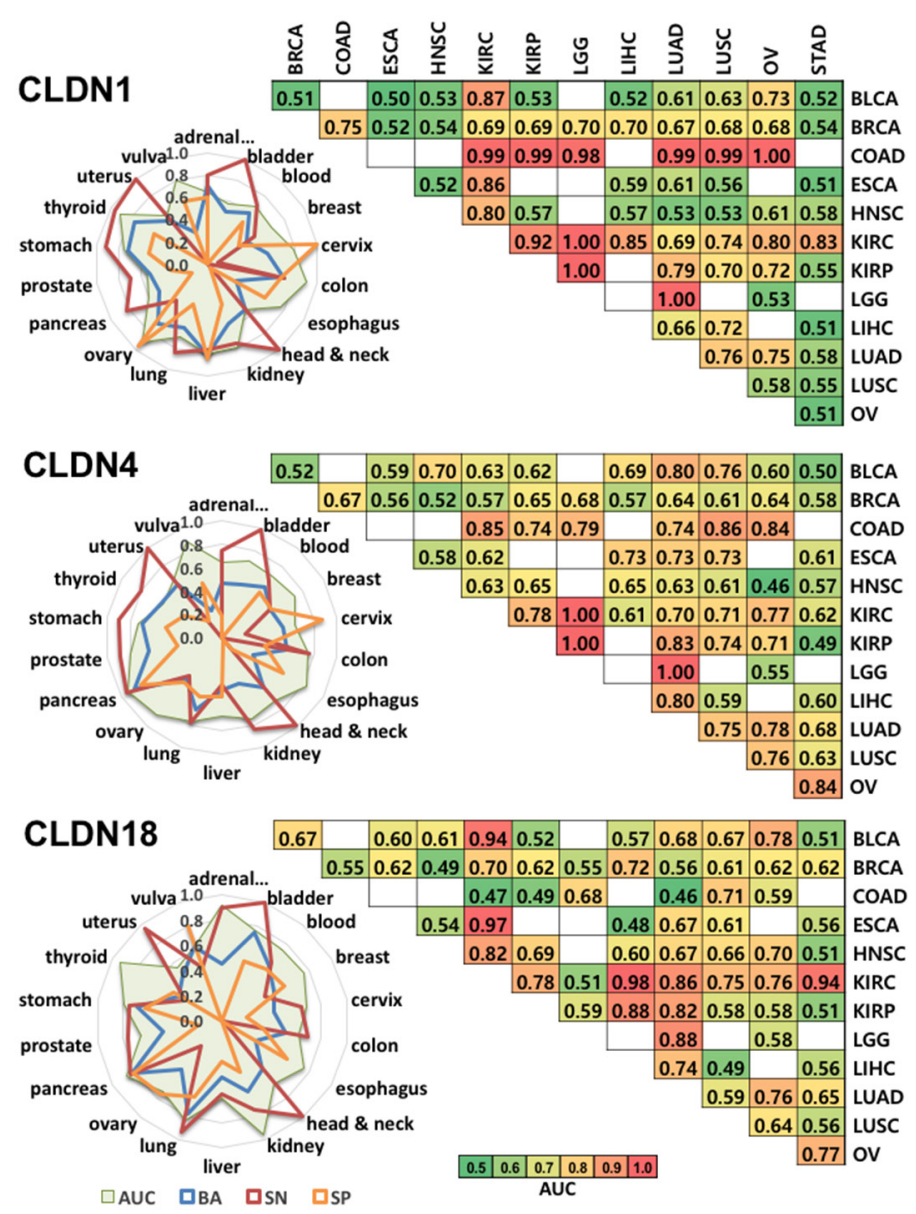

Figure 2: This figure shows CANES evaluation of expression of CLDN1, CLDN4, and CLDN18, as individual biomarkers for discriminating multiple cancer types. Left panel is a radial plot representing the predictive value (AUC, BA, SN and SP) of an expressed marker across 18 tumor types within the CANES database. The radial plot shows numeric magnitudes of performance values $(0$ to 1 ) from the centers of the circles in 18 radial directions corresponding to the 18 tumor types. In each radial plot, the green area represents $\mathrm{AUC}$, the blue line represents BA, the red line represents SN, and the orange line is SP. For example, the SN for CLDN4 in bladder cancer is 1.0. The right panel (matrix) represents how well each marker can distinguish between two cancer types. Heatmap color represents the level of AUC. The higher AUC in a heatmap cell indicates that the two pairwise-compared cancer types are better distinguished by a given marker. BLCA, bladder urothelial carcinoma; BRCA, breast invasive carcinoma; COAD, colon adenocarcinoma; ESCA, esophageal carcinoma; HNSC, head and neck squamous cell carcinoma; KIRC, kidney renal clear cell carcinoma; KIRP, kidney renal papillary cell carcinoma; LGG, brain lower grade glioma; LIHC, liver hepatocellular carcinoma; LUAD, lung adenocarcinoma; LUSC, lung squamous cell carcinoma; OV, ovarian serous cystadenocarcinoma. *White box: dataset not yet provided by TCGA. 
will also provide analysis to implement within translational research, improving the characterization of specific cancer types, identifying cancer progression pathways, and improving evidence-based biomarker therapeutic development.

Unlike other diseases, the development of clinical cancer biomarkers has been fraught with difficulties $[31,35]$. Despite several thousands of publications, the actual number of clinically approved biomarkers remains less than 100 [3, 30]. For general population screening, the prostate-specific antigen (PSA) remains the only approved serum biomarker, and guidelines even for its use have spurred controversy (e.g. men over age 40 vs. 50 , etc., non-family prostate cancer history, etc.), due to its high false positive rate, and subsequently, unnecessary, invasive procedures [36]. Similarly, while tumor whole genome and transcriptome sequencing have ushered in the advent of "personalized" therapies, individualized for specific patients, the cost/benefit of these massive analyses remains debatable, and these approaches may be confounded by uncontrolled false discovery rates and the genomic instability and heterogeneity found in most tumors [37]. Likewise, while the clinical utility of tumor-specific prognostic gene expression "signatures" has gained greater acceptance [5], many have not yet proved unreliable [38]. Even for well-known prognostic biomarkers, such as the Cancer Embryonic Antigen (CEA, colon cancer), CA-125 (ovarian cancer), and CA-19-9 (pancreatic cancer), their precise role(s) in the progression of those diseases remains largely unknown [30]. Moreover, the poor "bench-to-bedside" progression of preclinically discovered biomarkers has been attributed to a number of factors, including biased or low-rigor statistical assessment, irreproducibility, and an overall decreased quality of preclinical studies [39]. Despite a number of ambitious attempts to remedy these shortcomings [2, 32, 40], this overall trend has largely persisted [30, 31, 35].

\section{DISCUSSION}

One possible solution to increasing biomarker success rates is through the use of bioinformatics and improved statistical evaluation, using publically accessible databanks, thus increasing sample sizes and removing various confounding variables [3, 41, 42]. In this study, we undertook such an approach by designing
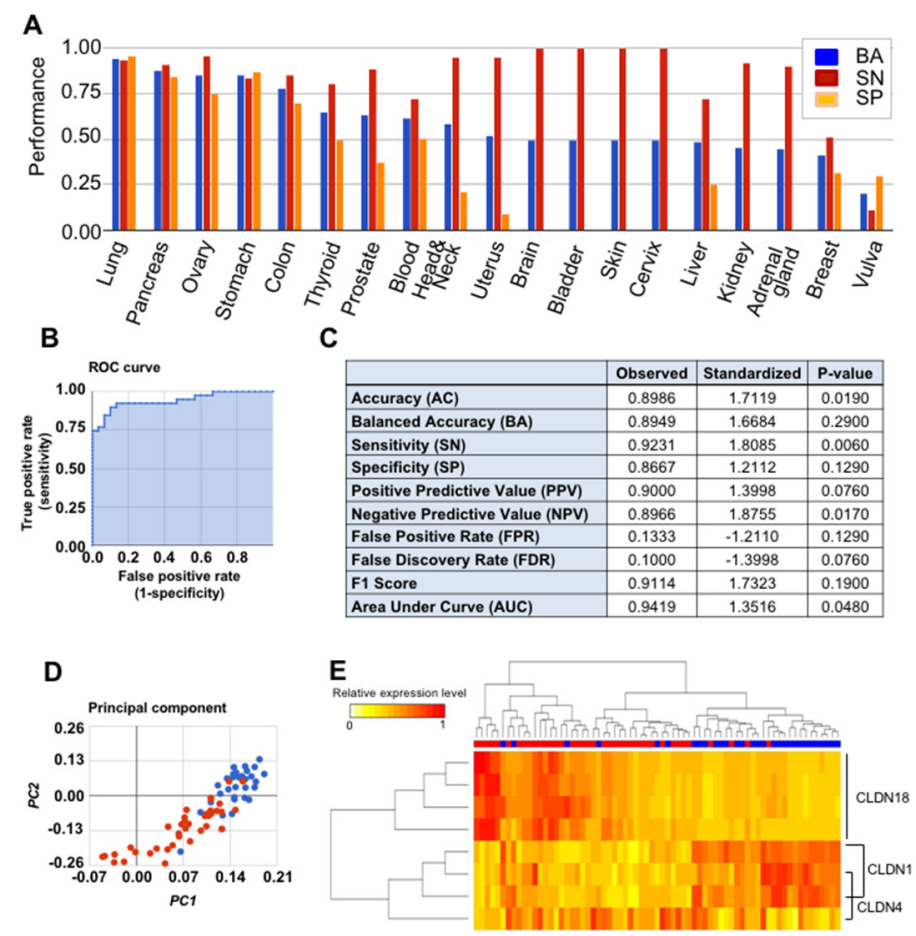

\begin{abstract}
Figure 3: This figure demonstrates CANES evaluation of the performance of a three-member $C L D N s$ 1/4/18 biomarker panel. (A) Balanced accuracy ("BA") (in blue), sensitivity ("SN") (in red), and specificity ("SP") (in orange) of the marker panel among 18 different cancer types. (B) Receiver operator characteristic (ROC) curve for the predictive accuracy of the panel in a TCGA gastric cancer dataset (GSE13911). AUC is the area under the ROC curve. The higher AUC indicates better performance in terms of both sensitivity and specificity. (C) Ten separate evaluation measures of the CLDN1/CLDN4/CLDN18 panel for gastric cancer dataset (GSE13911). (D) Principal components (PC) analysis (cancerous gastric tissues, red circles; normal gastric tissues, blue circles) showing the separation of cancerous and normal tissues along the first principal component (PC1) and second PC (PC2) in gastric cancer dataset GSE13911. (E) Heatmap cluster analysis showing the panel to clearly delineate cancerous vs. normal gastric tissues. The rows are genes or probes, and the columns are cancerous (red horizontal sidebars) and normal tissues (blue horizontal sidebars).
\end{abstract}


a single/multi-biomarker evaluation tool, CANES, a simple and user-friendly web-based application. CANES evaluates multiple markers, using the abovedescribed data repositories, to harness the power of "Big Data" for researchers to develop new models of translational research, for diagnostic and prognostic applications, and "targeted" therapies. By incorporating clinical data from those databases, matched to specific patient transcriptomes/genomes, CANES can evaluate the performance of multiple biomarkers for a number of clinical parameters, e.g., diagnosis, therapy response, survival, etc. (in contrast to other widely used analysis tools), thus increasing the robustness of assessment (for improved screening) and improving the probability of eventual clinical translation [30, 31]. While CANES continues to use all publicly available microarray datasets, it can also incorporate next generation sequencing technology (e.g., RNA-seq) datasets, for specific cancers, that are now increasingly available from the TCGA [10] and other databases.

Currently, CANES provides classification evaluation of numerous organ-based cancer types, including liver, lung, and pancreatic cancers, and many others. For each cancer subtype, even though there is considerable publically available expression data, with subtype information, CANES cannot yet support subtype-based classification evaluation, due to subtype's term diversity and lack of standardization of subtype terms. However, we have now designed a plan to update our system for subtype-based classification evaluation.

In summary, CANES is a powerful tool that will enable preclinical researchers to assist bench-side researchers in exploring available data for the disease of interest, as well as cater to the needs of bedside practitioners, to develop and implement cancer-specific biomarker therapies.

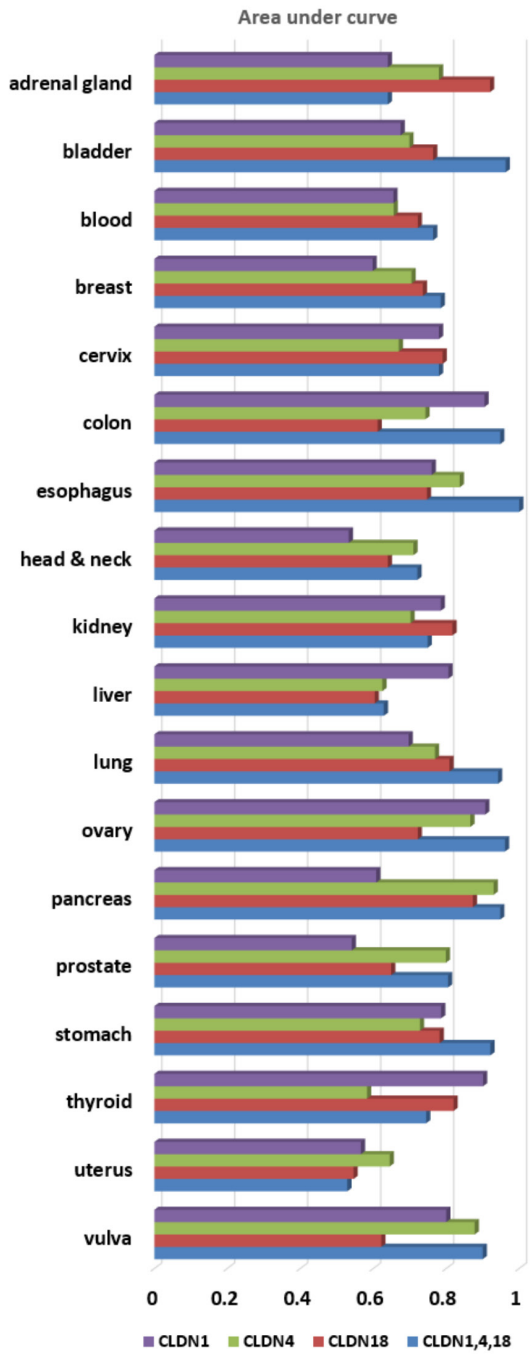

Figure 4: This figure shows a bar plot for diagnostic performances of three single-markers (CLDN1, $C L D N 4$ and $C L D N 18)$ and a multi-marker (CLDN1, CLDN4 and $C L D N 18)$ in terms of AUC across 18 tumor types. 
Table 2: Comparison of 5 biomarkers identified as Herceptin non-responsive biomarker between CANES vs. IPAbiomarker and Oncomine V4.5

\begin{tabular}{|c|c|c|c|c|c|c|c|c|c|}
\hline & \multicolumn{3}{|c|}{ CANES } & \multicolumn{3}{|c|}{ IPA-biomarker } & \multicolumn{3}{|c|}{ Oncomine v4.5 } \\
\hline & $\begin{array}{c}\text { Single } \\
\text { biomarker } \\
\text { evaluation } \\
\left(A C^{1}\right)\end{array}$ & $\begin{array}{c}\text { Multi-gene } \\
\text { biomarker } \\
\text { evaluation } \\
\left(\mathrm{AUC}^{1}\right)\end{array}$ & $\begin{array}{c}\text { Quantification } \\
\text { of single/ } \\
\text { multi-gene } \\
\text { biomarker }\end{array}$ & $\begin{array}{c}\text { Single } \\
\text { biomarker } \\
\text { evaluation }\end{array}$ & $\begin{array}{l}\text { Multi-gene } \\
\text { biomarker } \\
\text { evaluation }^{2}\end{array}$ & $\begin{array}{c}\text { Quantification } \\
\text { of single/ } \\
\text { multi-gene } \\
\text { biomarker }\end{array}$ & $\begin{array}{c}\text { Single gene } \\
\text { evaluation: } \\
\text { detection rate } \\
\left(\mathrm{A} / \mathrm{B}^{3}\right)\end{array}$ & $\begin{array}{l}\text { Multi-gene } \\
\text { biomarker } \\
\text { evaluation }{ }^{2}\end{array}$ & $\begin{array}{l}\text { Quantification of } \\
\text { single/multi-gene } \\
\text { biomarker }\end{array}$ \\
\hline ENAH & 0.826 & 0.854 & $\begin{array}{c}\text { Yes (11 } \\
\text { measurements) }\end{array}$ & Yes & - & No & $11.32 \%(6 / 53)$ & - & $\begin{array}{l}\text { Only single } \\
\text { biomarker } \\
\text { with limited } \\
\text { quantification }\end{array}$ \\
\hline RAD51 & 0.777 & & & Yes & & & $9.09 \%(4 / 44)$ & & \\
\hline ATF4 & 0.688 & 0.78 & & No & - & & $1.96 \%(1 / 51)$ & - & \\
\hline CHEK2 & 0.681 & & & No & & & $2.33 \%(1 / 43)$ & & \\
\hline ICOSLG & 0.653 & & & No & & & $0 \%$ & & \\
\hline
\end{tabular}

Previously, we proposed the five genes ENAH, RAD51, CHEK2, ATF4, and ICOSLG as Herceptin non-responsive biomarker candidates in breast cancer. Setting the genes as a reference set in breast cancer, we inspected the agreement between the five genes and each tool's results. IPA-biomarker does not report a numerical representation for evaluation except either detection or no detection. Oncomine reports the number of significant differential analyses relating to each candidate, without performance evaluation information. For comparison of multi-gene biomarker evaluation, we used the two multi-gene biomarker sets (one for ENAH and RAD51; the other for ATF4, CHEK2, and ICOSLG).

${ }^{1}$ AUC: average of area under curve.

${ }^{2}$ IPA and Oncomine do not support a multiple-gene biomarker evaluation (NA: non applicable).

${ }^{3}$ In breast cancer, "A" represents the number of total analyses relating to a given gene, and "B" the number of significant analyses relating to the gene. For example, in $E N A H$, Oncomine reports the 53 analyses relating to $E N A H$ in breast cancer. Out of them, six analyses reports the significant expression difference between breast cancer and normal groups.

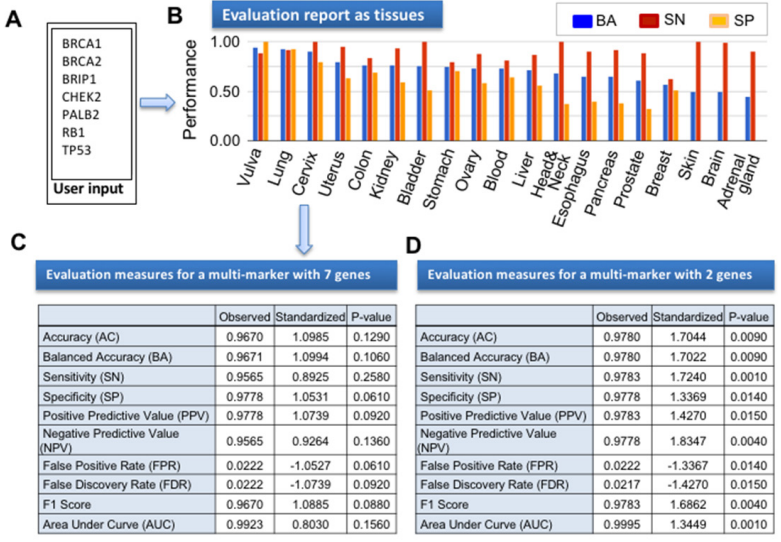

Figure 5: The figure here shows an example CANES output report following upload of breast cancer biomarker set. (A) In this instance, seven well-known breast cancer biomarkers (BRCA1, BRCA2, BRIP1, CHEK2, PALB2, RB1, and TP53) were evaluated for numerous performance measures in lung cancer. (B) Bar graph of three evaluation measures (BA, SN, and SP) of the 7-member panel across 18 organs. (C) The numerical evaluation measures of the 7-member panel in a lung cancer dataset (GSE18842). (D) The evaluation measures for a multi-marker with BRIP1 and RB1 in GSE18842. 

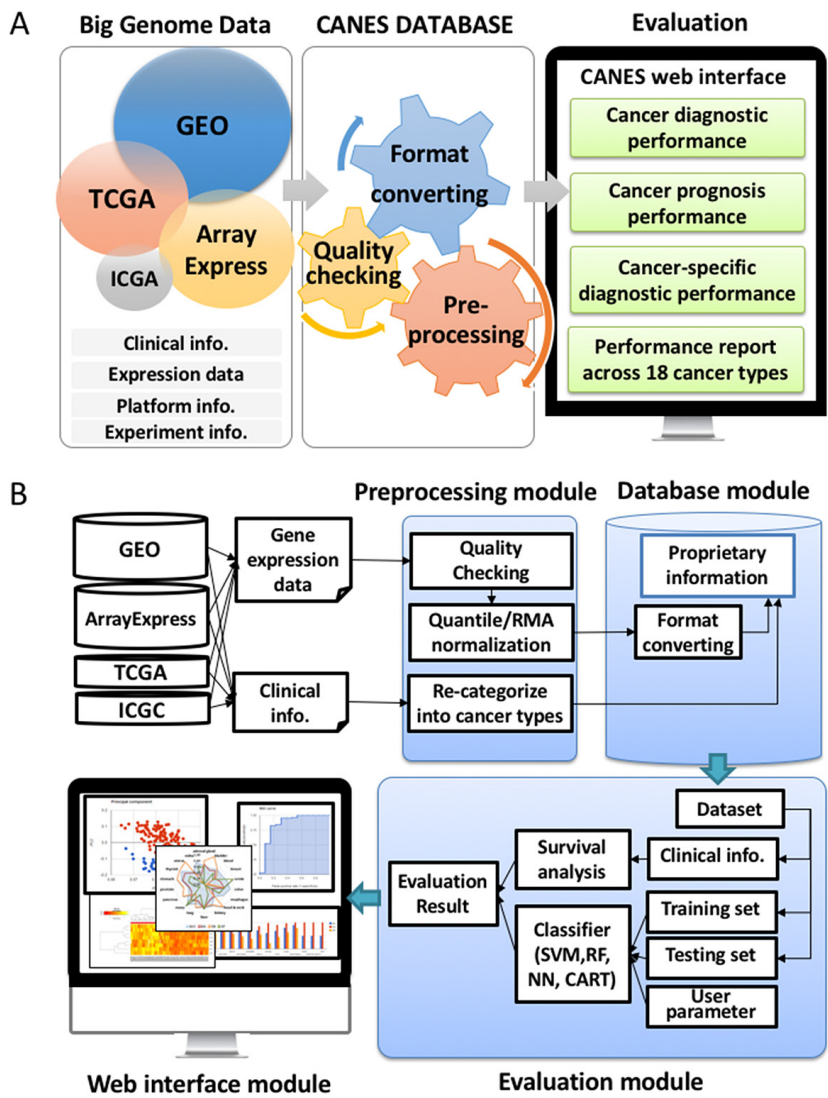

Figure 6: Key features of CANES. (A) The figure depicts how the web-based CANES tool provides a highly stringent evaluation system to compare user input marker candidates to datasets retrieved from four "Big Data" depository (TCGA, GEO, ArrayExpress, ICGC). For reproducibility of evaluation, CANES not only constructs its own preprocessed expression datasets but also provides various evaluation analyses (cancer diagnosis and prognosis). (B) For user inquires, CANES consists of four modules (1) a preprocessing module (a step for data normalization, as well as for clinical information summary); (2) a database module (for storing pre-calculated data); (3) an evaluation module (a step for evaluating users' input genes based on survival significance and classification performance); and (4) a web-interface module (for user-friendly visualization).
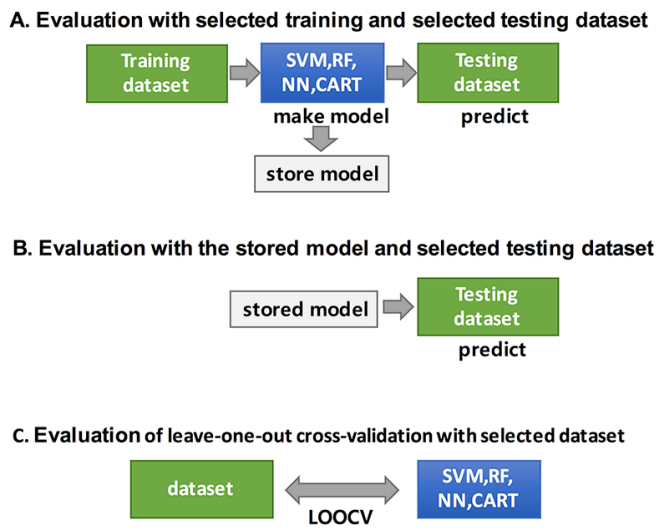

D. Evaluation with user-provided training dataset and selected testing dataset

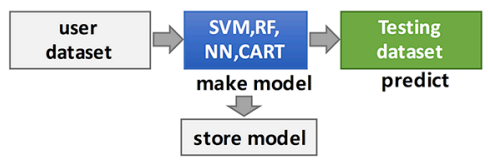

Figure 7: The figure shows how CANES performs the four classifications of the biomarker candidate evaluations. SVM, support vector machine; RF, random forest; NN, neural network; CART, a classification tree. Classifications are used to evaluate the performances power. 
Table 3: Comparison of databases relating to biomarker evaluation

\begin{tabular}{|c|c|c|c|c|c|}
\hline \multicolumn{2}{|l|}{ Functions } & \multirow{2}{*}{$\begin{array}{c}\text { CANES } \\
\text { Yes (Reporting } \\
\text { all the BAs and } \\
\text { AUCs across } \\
\text { pan-cancer) }\end{array}$} & \multirow{2}{*}{$\begin{array}{c}\text { ONCOMINE } \\
\text { Yes (reporting } \\
\text { the number } \\
\text { of over/under } \\
\text { expressed genes } \\
\text { in pan-cancer) }\end{array}$} & \multirow{2}{*}{$\begin{array}{c}\text { IPA-biomarker } \\
\text { No }\end{array}$} & \multirow{2}{*}{$\begin{array}{c}\text { cBioPortal } \\
\text { Yes (only } \\
\text { proportion of } \\
\text { alterations only } \\
\text { applied across } \\
\text { pan-cancer) }\end{array}$} \\
\hline $\begin{array}{l}\text { Performance } \\
\text { evaluation for single } \\
\text { marker }\end{array}$ & $\begin{array}{l}\text { Pan-cancer summary } \\
\text { view }\end{array}$ & & & & \\
\hline & Survival analysis & Yes & No & No & Yes \\
\hline & Utility for miRNA & Yes & No & Yes & No \\
\hline \multirow[t]{3}{*}{$\begin{array}{l}\text { Performance } \\
\text { evaluation for } \\
\text { multiple markers }\end{array}$} & $\begin{array}{l}\text { Pan-cancer summary } \\
\text { view }\end{array}$ & Yes & No & No & No \\
\hline & Survival analysis & Yes & No & No & No \\
\hline & Utility for miRNA & Yes & No & No & No \\
\hline Accessibility & & Free & $\begin{array}{c}\text { Free for } \\
\text { non-profit } \\
\text { organisations but } \\
\text { allow restricted } \\
\text { use }\end{array}$ & Commercial & Free \\
\hline \multicolumn{2}{|c|}{ User dataset is mandatory? } & No & No & Yes & No \\
\hline
\end{tabular}

\section{MATERIALS AND METHODS}

\section{Web-based $C A N$ cer-specific single/multi- biomarker Evaluation System (CANES)}

CANES postulates that biomarker candidates are well reproduced in multiple, independent datasets, regardless of different technology platforms (e.g., RTPCR, microarrays, RNA-Seq, etc.). CANES processes individual datasets in a preprocessing step (without merging all the datasets into a single pool). The evaluation phase then inspects whether or not the biomarker candidates are reproduced across multiple samples. CANES collects RNA molecular profiles from public databases and assigns them into distinct tumor types using their annotations, following a rigorous quality control process. CANES then provides evaluation results for userspecified, multiple markers, across various cancer types or studies. As shown in Figure 6, CANES has four modules: a preprocessing module, a database module, an evaluation module, and a web-interface module. The preprocessing module normalizes individual datasets separately for storage in the CANES internal database, which is then used as an expression resource for evaluation. For the selected biomarker candidate (single and/or multi-gene), the evaluation module provides numerous measures for assessing prediction performance.

Figure 6A shows a schematic of CANES' overall procedure. The CANES database draws information, from 18 distinct tumor type datasets, from "big genome" data depositories, including the Gene Expression Omnibus
(GEO) [44], TCGA [10], the International Cancer Genome Consortium (ICGC) [45, 46], and ArrayExpress, a functional genome database administered by the European Bioinformatics Institute [43]. After steps involving quality checking, format conversion (to match the user's biomarker search entry(ies)), and preprocessing, CANEs evaluates the performance of single/multi-biomarker candidates, based on four established classification methods (Figure 7A-7D).

CANES evaluation can also be based on a stored model, trained by various classifiers, to assess the predictive value of a test dataset (Figure 7B). Alternatively, the user can provide his/her own training dataset to train a model using the same four classifiers (Figure 7D). For classification, CANES employs a consensus of four different approaches, support vector machine (SVM), random forest (RF), neural networks (NN), and classification and regression trees (CART). For validation of user-provided datasets, CANES uses leave-one-out cross-validation (LOOCV) (Figure 7C).

Figure $6 \mathrm{~B}$ depicts a flowchart to further illustrate the CANES pipeline. The user uploads a marker candidate set through a web-interface module, entering the candidate set that is evaluated against clinical, preprocessed, and normalized gene expression data, that is then recategorized from the four above-mentioned public data repositories (Figure 6B). Based on user-defined cut-off levels for high vs. low gene expression, CANES evaluates the biomarker(s) for the following measures: AUC (an accuracy measurement based on the true positive rate plotted as a function of the false negative rate) [42], accuracy (AC), BA (defined as the arithmetic mean of SN 
and SP that corrects for imbalanced performance by the classifiers) [47], SN, SP, positive predictive value (PPV), negative predictive value (NPV), false positive rate (FPR), false discovery rate (FDR), and F1 score (a test accuracy measurement that considers both precision and recall) (Supplementary Table 1) [47].

Table 3 shows the notable advantages of CANES over other widely used biomarker database analyses, including Oncomine [48], IPA-Biomarker (www.qiagen. com/ingenuity), and cBioPortal [49], in terms of biomarker evaluation functionality. These include: 1) survival analysis, including Kaplan-Meier analysis and Cox proportional hazard regression; 2) evaluation of mature miRNAs as markers, as well as genes, for user-defined diagnostic or prognostic purposes; and 3) provision of a pan-cancer summary view for evaluating each single marker (Table 3).

\section{Preprocessing module}

The current version of CANES uses microarray data obtained from two public repositories and two cancer consortia, GEO [44], ArrayExpress [43], TCGA [10], and ICGC [46]. All expression datasets can be collected using the R package GEOquery [50]. In the CANES preprocessing module, expression datasets from these public repositories are parsed and normalized by quantiles robust multi-array average (RMA) [51]. For RNA sequencing data, counts are normalized to expression values. All datasets with missing rates $>5 \%$ are excluded, and the remaining datasets with missing values are imputed by the 'impute' package [52] of Bioconductor. To detect outliers caused by instrument error or sample contamination, mislabeling, or misprocessing, we use within-group and between-group correlations [53]. Since all detected outlier samples are marked, users can exclude them from their analyses, using specified options. Moreover, available clinical information and sample annotations are parsed into the CANES database. To define the exact cancer type or subtype, we confirm or recategorize diagnoses, prognoses, and drug responses as designs of the dataset. All processed expression data are converted into customized, (Figure 6B, middle) indexed binary files for fast retrieval as big genomic expression data. The preprocessing module is implemented using Python and R.

\section{Database module}

The database module contains preprocessed expression datasets and their corresponding annotation data. Currently, the preprocessed datasets consist of gene expression data and annotation data for 94,147 samples (Supplementary Table 2). Gene expression data are obtained from these samples with broadly used gene expression microarray platforms and RNA sequencing platforms, and processed as described. The database module is implemented using MySQL and Python. All expression data is saved as customized indexed binary files.

\section{Evaluation module}

The evaluation module of CANES is implemented using classification methods such as SVM, RF, NN, and CART. For user-selected multiple markers, this module provides the evaluation result with the evaluation measures across 18 cancer-types, based on ten evaluation measures, including AUC, AC, BA, SN, SP, PPV, NPV, FPR, FDR, and F1 score [47] (Supplementary Table 1). In addition, the evaluation module provides standardized evaluation measures and empirical p-values to address the problem of randomly chosen marker sets, with a large number of probes, tending to show good performance (for further description in "Summary evaluation measurements and their p-value calculations in CANES" in the Materials and Methods section). To measure the contribution of a single marker to the performance of multi-markers, CANES provides an influence measure, which is the difference between the evaluation measure for all markers and that for all markers excluding the single marker. All these manipulations can be conducted by four different evaluation schemes, as follows (Figure 6B).

i) Evaluation of multiple markers with selected training and testing datasets. CANES can conduct prediction analysis using specific cancer types or studies. Users can generate and store the prediction model for their own multi-marker lists using the selected dataset and classification method. Graphical and interactive result layouts are provided and can be saved.

ii) Evaluation using a stored prediction model on a selected testing dataset. CANES stores the evaluation result, which can then be used on a different testing dataset. For example, users can store the prediction model with breast cancer markers and breast cancer datasets, and then evaluate this stored model against a liver cancer dataset.

iii) Evaluation of LOOCV with the selected dataset. To prevent overfitting by any specific training dataset, CANES can evaluate multiple markers using LOOCV. In this evaluation module, CANES can also support the evaluation of individual markers, in a multi-marker set, by measuring the contribution level of the performance of multi-markers.

iv) Evaluation with the user-provided training dataset and the selected testing dataset. CANES allows evaluation of a prediction model generated by a userprovided dataset. The user dataset is uploaded via a web-interface module, is preprocessed and normalized, and is then used as a training dataset employingdifferent classification methods. The prediction models trained with the user's own dataset are tested with independent datasets from public repositories.

\section{Web-interface module}

The web-interface of CANES consists of the input layout and the result explorer. The input layout is the interface 
that transfers user-selected multiple markers and queried parameters to the evaluation module. In the input layout, a user can input a set of official gene symbols, miRNAs, or probe IDs, and select either a preprocessed public dataset, or a user-uploaded private dataset, as the training dataset. The result explorer provides tables and graphical visualization of the evaluation results (Figure 6B, bottom left). The CANES web-interface module is implemented using PHP, within a JQuery and CodeIgniter framework.

CANES is freely accessible from the CANES website http://bibs.snu.ac.kr/software/canes. Moreover, the design and implementation of CANES facilitates easy incorporation of additional query functions and applications, as well as other datasets, irrespective of cancer type, in the form of pre-processed datasets. All evaluation results are presented in a table and/or graphical visualization, and can be downloaded as high-quality PDF images and CSV-based text-format spreadsheets.

\section{Summary evaluation measurements, and their p-value calculations, in CANES}

We defined various evaluation measurements, in consistency with widely accepted formulas, as follows: $\operatorname{accuracy}(\mathrm{AC})$ is $(\mathrm{TP}+\mathrm{TN}) /(\mathrm{TP}+\mathrm{TN}+\mathrm{FP}+\mathrm{FN})$ where $\mathrm{TP}$ is the true positive value, TN the true negative, FP the false positive, and FN the false negative value. Sensitivity ( $\mathrm{SN}$ ) is defined as $\mathrm{TP} /(\mathrm{TP}+\mathrm{FN})$, and specificity (SP) by $\mathrm{TN} /(\mathrm{FP}+\mathrm{TN})$. In addition, balanced accuracy (BA) is defined as $(\mathrm{SN}+\mathrm{SP}) / 2$, while positive predictive value (PPV) is defined as $\mathrm{TP} /(\mathrm{TP}+\mathrm{FP})$, negative predictive value (NPV) as $\mathrm{TN} /(\mathrm{FN}+\mathrm{TN})$, false discovery rate (FDR) as 1-PPV, and F1 as $2 \mathrm{TP} /(2 \mathrm{TP}+\mathrm{FP}+\mathrm{FN})$. Area under the curve (AUC) is the area under the receiver operating characteristic (ROC) curve, which is the line representing the true positive rate (TPR or sensitivity) and false positive rate (FPR or 1-specificity) of any distinct diagnostic test. AUC can also be used as an index of the test's performance (Supplementary Table 1).

Based on these evaluation measures, CANES provides standardized evaluation measures, and empirical p-values, as follows:

i) CANES calculates the observed evaluation measure $\left(t_{i, o}\right)$ for the user-defined marker set with $m_{i}$ probes for the $i_{t h}$ dataset.

ii) CANES uses a preconstructed empirical null distribution of the observed evaluation measure $\left(t_{i, o}\right)$. In the $i_{\text {th }}$ dataset, $n$ probe sets are generated with randomly selected $m_{i}$ probes. The empirical null distribution is then constructed using the evaluation measure $\left(t_{i, r}\right)$ for the $n$ probe sets.

iii) Using the empirical null distribution, the average $\left(\bar{t}_{i}\right)$ and the standard deviation $\left(s_{i}\right)$ can be calculated as follows:

$$
\bar{t}_{i .}=\frac{\sum_{r=1}^{n} t_{i, r}}{n} \text { and } s_{i}=\sqrt{\frac{\sum_{r=1}^{n}\left(t_{i, r}-\bar{t}_{i .}\right)^{2}}{n-1}}
$$

The standardized evaluation measure $\left(Z_{i}\right)$, and its p-value, are defined as follows:

$$
z_{i}=\frac{t_{i, o}-\bar{t}_{i .}}{s_{i}} \text { and } p_{i}=\frac{\sum_{r=1}^{n} I\left(t_{i, r} \geq t_{i, o}\right)}{n}
$$

Note that $\bar{t}_{i}$ and $s_{i}$ need to be computed for $m_{i}$ probes in the $i_{t h}$ dataset.

There is an advantage in using $z_{i}$ over $t_{i, o}$. We found out that the values of $t_{i, o}$ tend to increase as the number of probes increases. Thus, $t_{i, o}$ needs to be standardized to be sufficiently robust to the number of probes. $z_{i}$ is a standardized version of $t_{i, o}$, and is a more appropriate evaluation measure than $t_{i, o}$. Through $z_{i}$, a direct comparison of the diagnostic performance between models with different numbers of probes becomes possible. The empirical p-value is the relative frequency that the randomly selected marker sets have better performances than the user-defined marker set. Note that these p-values are the same for $z_{i}$ and $t_{i, o}$.

To summarize performance measures from $k$ datasets into a single measure, CANES provides the summarized $\mathrm{p}$-value by combining $\mathrm{p}$-values as follows:

i) $\min P$ method $: P_{A}=\min \left\{p_{1}, p_{2}, \ldots, p_{k}\right\}$

ii) Fisher's method: $P_{A}=1-\chi_{2 k}^{2}\left(-2 \sum_{i=1}^{k} \ln \left(p_{i}\right)\right)$

iii) Stouffer's method :

$$
P_{A}=1-\Phi\left(\frac{\sum_{i=1}^{k} Z_{i}}{\sqrt{k}}\right) \text {, when }
$$
$Z=\Phi^{-1}\left(1-p_{i}\right)$

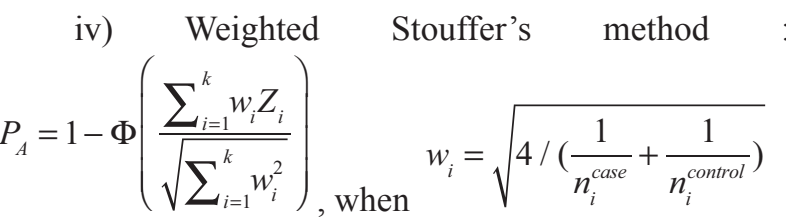

\section{System implementation of CANES}

The job scheduling scheme of CANES is based on a first-come, first-serve process. To support intensive queries from public users, the CANES system consists of one web-server and 10 Xeon $\AA$ (manufactured by Intel) calculation servers. Once a job is submitted by the user, the job is executed in the background, on calculation servers. Therefore, users don't need to keep the submission webpage open on their browser until the job is finished. After the user's job is done, CANES sends a notice email with a direct link to the results page. To prevent waste of computing resource by redundant model fitting, CANES can keep the previous search results in the cache space and provide the stored results, without re-evaluation. 


\section{Abbreviations}

AUC, area under curve; BA, balanced accuracy; CANES, CANcer-specific single/multi-biomarker Evaluation System; CART, classification and regression trees; CEA, Cancer Embryonic Antigen; EGFR, epidermal growth factor receptor; FDR, false discovery rate; FN, false negative value; FP, false positive value; FPR, false positive rate; GC, gastric cancer; GEO, Gene Expression Omnibus; ICGC, the International Cancer Genome Consortium; LOOCV, leave-one-out cross-validation; NN, neural networks; NPV, negative predictive value; PPV, positive predictive value; PSA, prostate-specific antigen; REMARK, REporting recommendations for tumor MARKers; RF, random forest; RMA, robust multi-array average; ROC, receiver operating characteristic; SN, sensitivity; SP, specificity; STRING, Search Tool for the Retrieval of Interacting Genes/Proteins; SVM, support vector machine; TCGA, The Cancer Genome Atlas; TN, true negative value; TP, true positive value.

\section{Author contributions}

M-S.K, Y.H.K, and T.P conceived the idea, designed the study, and contributed to writing the manuscript. M-S.K and S.L implemented CANES and analysed the resulting data. Y.Z.A, H.R.C, and S.N wrote parts of the manuscript. S.N and Y.Z.A performed the analysis for single biomarker evaluation of Claudin family gene expression. H.R.C, and Y.H.K conducted and analysed tissue microarrays and immunohistochemistry from gastric cancer patient samples. All authors discussed the results and their presentation. All authors commented on and edited the manuscript.

\section{ACKNOWLEDGMENTS}

We would like to thank Curt Balch for critical reading and preparation of the manuscript.

\section{CONFLICTS OF INTEREST}

The authors declare that there are no conflicts of interest.

\section{FUNDING}

This work was supported by the Bio-Synergy Research Project (2013M3A9C4078158) of the Ministry of Science, ICT and Future Planning through the National Research Foundation, the Republic of Korea, and by grants of the Korea Health Technology R\&D Project through the Korea Health IndustryDevelopment Institute (KHIDI), funded by the Ministry of Health \& Welfare, Republic of Korea (HI15C2165, HI16C2037) (to T.P.); by grants of National Research Foundation of Korea (MSIP) 2015R1A2A1A10052661 (to YHK), and Basic Science Research Program through the National Research Foundation of Korea (NRF) funded by the Ministry of Education (2016R1D1A1B03933145) (to S.N.)

\section{REFERENCES}

1. Kern SE. Why your new cancer biomarker may never work: recurrent patterns and remarkable diversity in biomarker failures. Cancer Res. 2012; 72: 6097-101. https://doi. org/10.1158/0008-5472.CAN-12-3232.

2. Altman DG, McShane LM, Sauerbrei W, Taube SE. Reporting Recommendations for Tumor Marker Prognostic Studies (REMARK): explanation and elaboration. PLoS Med. 2012; 9: e1001216. https://doi.org/10.1371/journal. pmed.1001216.

3. Drucker E, Krapfenbauer K. Pitfalls and limitations in translation from biomarker discovery to clinical utility in predictive and personalised medicine. EPMA J. 2013; 4: 7. https://doi.org/10.1186/1878-5085-4-7.

4. Bast RC Jr. Status of tumor markers in ovarian cancer screening. J Clin Oncol. 2003; 21: 200s-5s. https://doi. org/10.1200/JCO.2003.01.068.

5. Bourzac K. Diagnosis: early warning system. Nature. 2014; 513: S4-6. https://doi.org/10.1038/513S4a.

6. Liu C, Liu A, Halabi S. A min-max combination of biomarkers to improve diagnostic accuracy. Stat Med. 2011; 30: 2005-14. https://doi.org/10.1002/sim.4238.

7. Langley RR, Fidler IJ. Tumor cell-organ microenvironment interactions in the pathogenesis of cancer metastasis. Endocr Rev. 2007; 28: 297-321. https://doi.org/10.1210/ er.2006-0027.

8. Visvader JE. Cells of origin in cancer. Nature. 2011; 469: 314-22. https://doi.org/10.1038/nature09781.

9. Kandoth C, McLellan MD, Vandin F, Ye K, Niu B, Lu C, Xie M, Zhang Q, McMichael JF, Wyczalkowski MA, Leiserson MD, Miller CA, Welch JS, et al. Mutational landscape and significance across 12 major cancer types. Nature. 2013; 502: 333-9. https://doi.org/10.1038/ nature 12634

10. Cancer Genome Atlas Research Network, Weinstein JN, Collisson EA, Mills GB, Shaw KR, Ozenberger BA, Ellrott K, Shmulevich I, Sander C, Stuart JM. The Cancer Genome Atlas Pan-Cancer analysis project. Nat Genet. 2013; 45: 1113-20. https://doi.org/10.1038/ng.2764.

11. Steins M, Thomas M, Geissler M. Erlotinib. Recent Results Cancer Res. 2014; 201: 109-23. https://doi. org/10.1007/978-3-642-54490-3_6.

12. Nam S, Chang HR, Kim KT, Kook MC, Hong D, Kwon CH, Jung HR, Park HS, Powis G, Liang H, Park T, Kim YH. PATHOME: an algorithm for accurately detecting differentially expressed subpathways. Oncogene. 2014; 33: 4941-51. https://doi.org/10.1038/onc.2014.80. 
13. Chang H, Nam S, Kook MC, Kim KT, Liu X, Yao H, Jung H, Lemos R, Seo H, Park H, Gim Y, Hong D, Huh I, et al. HNF4alpha is therapeutic target that links AMPK to WNT signaling in early-stage gastric cancer. Gut. 2016; 65: 19-32. https://doi.org/10.1136/gutjnl-2014-307918.

14. Franceschini A, Szklarczyk D, Frankild S, Kuhn M, Simonovic M, Roth A, Lin J, Minguez P, Bork P, von Mering C, Jensen LJ. STRING v9.1: protein-protein interaction networks, with increased coverage and integration. Nucleic Acids Res. 2013; 41: D808-15. https://doi.org/10.1093/nar/ gks1094.

15. Eftang LL, Esbensen Y, Tannaes TM, Blom GP, Bukholm IR, Bukholm G. Up-regulation of CLDN1 in gastric cancer is correlated with reduced survival. BMC Cancer. 2013; 13: 586. https://doi.org/10.1186/1471-2407-13-586.

16. Kwon MJ, Kim SH, Jeong HM, Jung HS, Kim SS, Lee JE, Gye MC, Erkin OC, Koh SS, Choi YL, Park CK, Shin YK. Claudin-4 overexpression is associated with epigenetic derepression in gastric carcinoma. Lab Invest. 2011; 91: 1652-67. https://doi.org/10.1038/ labinvest.2011.117.

17. Oshima T, Shan J, Okugawa T, Chen X, Hori K, Tomita T, Fukui H, Watari J, Miwa H. Down-regulation of claudin-18 is associated with the proliferative and invasive potential of gastric cancer at the invasive front. PLoS One. 2013; 8: e74757. https://doi.org/10.1371/journal.pone.0074757.

18. Resnick MB, Gavilanez M, Newton E, Konkin T, Bhattacharya B, Britt DE, Sabo E, Moss SF. Claudin expression in gastric adenocarcinomas: a tissue microarray study with prognostic correlation. Hum Pathol. 2005; 36: 886-92. https://doi.org/10.1016/j.humpath.2005.05.019.

19. Sanada Y, Oue N, Mitani Y, Yoshida K, Nakayama H, Yasui W. Down-regulation of the claudin-18 gene, identified through serial analysis of gene expression data analysis, in gastric cancer with an intestinal phenotype. J Pathol. 2006; 208: 633-42. https://doi.org/10.1002/path.1922.

20. Zhu JL, Gao P, Wang ZN, Song YX, Li AL, Xu YY, Wang MX, Xu HM. Clinicopathological significance of claudin-4 in gastric carcinoma. World J Surg Oncol. 2013; 11: 150. https://doi.org/10.1186/1477-7819-11-150.

21. Melchor L, Benitez J. The complex genetic landscape of familial breast cancer. Hum Genet. 2013; 132: 845-63. https://doi.org/10.1007/s00439-013-1299-y.

22. Malkin D, Li FP, Strong LC, Fraumeni JF Jr, Nelson CE, Kim DH, Kassel J, Gryka MA, Bischoff FZ, Tainsky MA, Friend SH. Germ line p53 mutations in a familial syndrome of breast cancer, sarcomas, and other neoplasms. Science. 1990; 250: 1233-8.

23. Sanchez-Palencia A, Gomez-Morales M, Gomez-Capilla JA, Pedraza V, Boyero L, Rosell R, Farez-Vidal ME. Gene expression profiling reveals novel biomarkers in nonsmall cell lung cancer. Int J Cancer. 2011; 129: 355-64. https:// doi.org/10.1002/ijc.25704.
24. Westfall PH, Young SS. (1993). Resampling-based multiple testing : examples and methods for P-value adjustment. (New York: Wiley).

25. Nam S, Chang HR, Jung HR, Gim Y, Kim NY, Grailhe R, Seo HR, Park HS, Balch C, Lee J, Park I, Jung SY, Jeong KC, et al. A pathway-based approach for identifying biomarkers of tumor progression to trastuzumab-resistant breast cancer. Cancer Lett. 2015; 356: 880-90. https://doi.org/10.1016/j. canlet.2014.10.038.

26. Jimenez-Marin A, Collado-Romero M, Ramirez-Boo M, Arce C, Garrido JJ. Biological pathway analysis by ArrayUnlock and Ingenuity Pathway Analysis. BMC Proc. 2009; 3: S6. https://doi.org/10.1186/1753-6561-3-S4-S6.

27. Ghosh S, Vivar JC, Sarzynski MA, Sung YJ, Timmons JA, Bouchard C, Rankinen T. Integrative pathway analysis of a genome-wide association study of $(\mathrm{V}) \mathrm{O}(2 \mathrm{max})$ response to exercise training. J Appl Physiol (1985). 2013; 115: 134359. https://doi.org/10.1152/japplphysiol.01487.2012.

28. Rhodes DR, Yu J, Shanker K, Deshpande N, Varambally R, Ghosh D, Barrette T, Pandey A, Chinnaiyan AM. ONCOMINE: a cancer microarray database and integrated data-mining platform. Neoplasia. 2004; 6: 1-6.

29. Hanauer DA, Rhodes DR, Sinha-Kumar C, Chinnaiyan AM. Bioinformatics approaches in the study of cancer. Curr Mol Med. 2007; 7: 133-41.

30. Diamandis EP. Cancer biomarkers: can we turn recent failures into success? J Natl Cancer Inst. 2010; 102: 14627. https://doi.org/10.1093/jnci/djq306.

31. Rhea JM, Molinaro RJ. Cancer biomarkers: surviving the journey from bench to bedside. MLO Med Lab Obs. 2011; 43: 10-2, 6, 8; quiz 20, 2.

32. Pepe MS, Etzioni R, Feng Z, Potter JD, Thompson ML, Thornquist M, Winget M, Yasui Y. Phases of biomarker development for early detection of cancer. J Natl Cancer Inst. 2001; 93: 1054-61.

33. Pesch B, Bruning T, Johnen G, Casjens S, Bonberg N, Taeger D, Muller A, Weber DG, Behrens T. Biomarker research with prospective study designs for the early detection of cancer. Biochim Biophys Acta. 2014; 1844: 874-83. https://doi.org/10.1016/j.bbapap.2013.12.007.

34. Yotsukura S, Mamitsuka H. Evaluation of serumbased cancer biomarkers: a brief review from a clinical and computational viewpoint. Crit Rev Oncol Hematol. 2015; 93: 103-15. https://doi.org/10.1016/j. critrevonc.2014.10.002.

35. Diamandis EP. The failure of protein cancer biomarkers to reach the clinic: why, and what can be done to address the problem? BMC Med. 2012; 10: 87. https://doi. org/10.1186/1741-7015-10-87.

36. Wilt TJ, Scardino PT, Carlsson SV, Basch E. Prostatespecific antigen screening in prostate cancer: perspectives on the evidence. J Natl Cancer Inst. 2014; 106: dju010. https://doi.org/10.1093/jnci/dju010. 
37. Burrell RA, McGranahan N, Bartek J, Swanton C. The causes and consequences of genetic heterogeneity in cancer evolution. Nature. 2013; 501: 338-45. https://doi.org/10.1038/ nature12625.

38. Subramanian J, Simon R. Gene expression-based prognostic signatures in lung cancer: ready for clinical use? J Natl Cancer Inst. 2010; 102: 464-74. https://doi. org/10.1093/jnci/djq025.

39. Begley CG, Ellis LM. Drug development: raise standards for preclinical cancer research. Nature. 2012; 483: 531-3. https://doi.org/10.1038/483531a.

40. Baron JA. Screening for cancer with molecular markers: progress comes with potential problems. Nat Rev Cancer. 2012; 12: 368-71. https://doi.org/10.1038/nrc3260.

41. Diamandis EP, Bast RC Jr, Gold P, Chu TM, Magnani JL. Reflection on the discovery of carcinoembryonic antigen, prostate-specific antigen, and cancer antigens CA125 and CA19-9. Clin Chem. 2013; 59: 22-31. https://doi. org/10.1373/clinchem.2012.187047.

42. Izmailov R, Guest PC, Bahn S, Schwarz E. Algorithm development for diagnostic biomarker assays. Int Rev Neurobiol. 2011; 101: 279-98. https://doi.org/10.1016/ B978-0-12-387718-5.00011-0.

43. Kolesnikov N, Hastings E, Keays M, Melnichuk O, Tang YA, Williams E, Dylag M, Kurbatova N, Brandizi M, Burdett T, Megy K, Pilicheva E, Rustici G, et al. ArrayExpress update-simplifying data submissions. Nucleic Acids Res. 2015; 43: D1113-6. https://doi. org/10.1093/nar/gku1057.

44. Barrett T, Edgar R. Gene expression omnibus: microarray data storage, submission, retrieval, and analysis. Methods Enzymol. 2006; 411: 352-69. https://doi. org/10.1016/S0076-6879(06)11019-8.

45. Barrett IP. Cancer genome analysis informatics. Methods Mol Biol. 2010; 628: 75-102. https://doi. org/10.1007/978-1-60327-367-1_5.
46. Jennings J, Hudson TJ. Reflections on the founding of the International Cancer Genome Consortium. Clin Chem. 2013; 59: 18-21. https://doi.org/10.1373/ clinchem.2012.184713.

47. Straube S, Krell MM. How to evaluate an agent's behavior to infrequent events?-Reliable performance estimation insensitive to class distribution. Front Comput Neurosci. 2014; 8: 43. https://doi.org/10.3389/fncom.2014.00043.

48. Rhodes DR, Kalyana-Sundaram S, Mahavisno V, Varambally R, Yu J, Briggs BB, Barrette TR, Anstet MJ, Kincead-Beal C, Kulkarni P, Varambally S, Ghosh D, Chinnaiyan AM. Oncomine 3.0: genes, pathways, and networks in a collection of 18,000 cancer gene expression profiles. Neoplasia. 2007; 9: 166-80.

49. Gao J, Aksoy BA, Dogrusoz U, Dresdner G, Gross B, Sumer SO, Sun Y, Jacobsen A, Sinha R, Larsson E, Cerami E, Sander C, Schultz N. Integrative analysis of complex cancer genomics and clinical profiles using the cBioPortal. Sci Signal. 2013; 6: pl1. https://doi.org/10.1126/ scisignal.2004088.

50. Davis S, Meltzer PS. GEOquery: a bridge between the Gene Expression Omnibus (GEO) and BioConductor. Bioinformatics. 2007; 23: 1846-7. https://doi.org/10.1093/ bioinformatics/btm 254 .

51. Bolstad BM, Irizarry RA, Astrand M, Speed TP. A comparison of normalization methods for high density oligonucleotide array data based on variance and bias. Bioinformatics. 2003; 19: 185-93.

52. Troyanskaya O, Cantor M, Sherlock G, Brown P, Hastie T, Tibshirani R, Botstein D, Altman RB. Missing value estimation methods for DNA microarrays. Bioinformatics. 2001; 17: 520-5.

53. Lee EK, Yi SG, Park T. arrayQCplot: software for checking the quality of microarray data. Bioinformatics. 2006; 22: 2305-7. https://doi.org/10.1093/bioinformatics/btl367. 